\title{
Frech, Siri; Scurrell, Babette; Willisch, Andreas (Hrsg.) (2017): Neuland gewinnen. Die Zukunft in Ostdeutschland gestalten
}

\author{
Berlin - Ch. Links Verlag. 271 S., 193 Abb.
}

Helga Kanning ${ }^{1}$ (D)

Online publiziert: 2. Januar 2018

๑) Springer-Verlag GmbH Deutschland, ein Teil von Springer Nature 2017

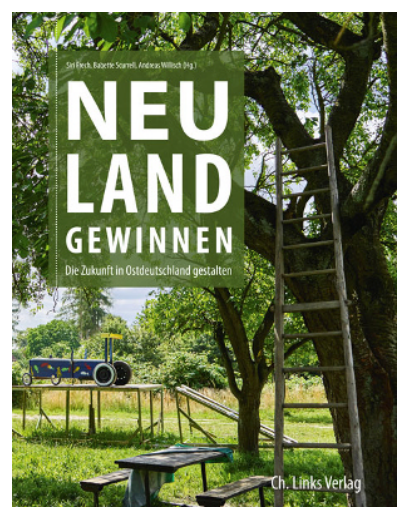

„Neuland gewinnen“ - der Titel des Buches ist gleichzeitig Programm: „Neulandgewinner. Zukunft erfinden vor Ort" heißt das Programm, mit dem die Robert Bosch Stiftung seit 2013 engagierte Menschen und Initiativen fördert und sie damit unterstützt, die Zukunft ländlicher Regionen in Ostdeutschland eigeninitiativ positiv zu gestalten (vgl. Links/Volke 2009). Mit individuellem Mentoring, Erfahrungsaustausch und Zugriff auf Expertisen in halbjährlichen Werkstätten sowie einer relativ kleinen finanziellen Unterstützung von durchschnittlich 50.000 Euro für zwei Jahre werden die „Neulandgewinner“ ermuntert, „Neuland zu betreten“, so der Leitsatz der Robert Bosch Stiftung, um die Lebensqualität in ihrer Umgebung mit innovativen Ideen zu verbessern. Die mehr als 1000 eingereichten Bewerbungen in den drei Ausschreibungsrunden bis 2017 dokumentieren das hohe zivilgesellschaftliche und kreative Potenzial der inzwischen mehr als 50 geförderten Projekte.

Apl. Prof. Dr.-Ing. Helga Kanning

kanning@umwelt.uni-hannover.de

1 Institut für Umweltplanung, Leibniz Universität Hannover, Herrenhäuser Straße 2, 30419 Hannover, Deutschland
Im Kontext der Transformations- und Transitionsforschung, die in Deutschland besonders mit dem Hauptgutachten „Welt im Wandel: Gesellschaftsvertrag für eine Große Transformation“ des Wissenschaftlichen Beirats Globale Umweltveränderungen (WBGU 2011) auch über die engere wissenschaftliche Diskussion hinaus populär geworden ist, lassen sich die „Neulandgewinner“ als „Pioniere des Wandels" und - spezifiziert mit dem Fokus auf die Gewinnung von Neuland bzw. neuer Räume als „Raumpioniere“ (Faber/Oswalt 2013) bezeichnen, wie dies auch die Herausgeber und Autoren der vorliegenden Fachpublikation tun. Die Neulandgewinner agieren als Raumpioniere, indem sie kreativ innovative Projekte für gesellschaftlichen Wandel in ländlichen Räumen umsetzen. Mit ihren innovativen Ideen transformieren sie Räume sowohl in materiell-physischer Hinsicht, z. B. durch Umnutzung von Gebäuden, als auch in kulturell-diskursiver Hinsicht, z. B. durch neue gemeinschaftliche Lebens- und Arbeitsformen, und gewinnen damit in beiderlei Hinsicht „Neuland“ im wahrsten Sinne des Wortes.

In diesem Kontext stellt auch das vorliegende Buch an sich eine Besonderheit dar. Wie es die Herausgeber in ihrem Vorwort schildern, ist die Idee dazu bei den Neulandgewinnern selbst entstanden, so dass deren Darstellungen auch den größten Raum einnehmen.

Mit einem einführenden Beitrag spannen die Herausgeber, die auch als Mentoren agieren, den gesellschafts- und raumbezogenen Kontext auf. Sie spüren dabei traditionellen Verständnissen von Land und Stadt sowie dem radikalen gesellschaftlichen Wandel im ländlichen Raum nach. Besonders sichtbar sind diese Veränderungen in Ostdeutschland. Weite Teile des ländlichen Raums dort sind weiterhin durch landwirtschaftliche Großbetriebe und industrielle Agrarwirtschaft geprägt, deren Gewinne größtenteils aus den Regionen abfließen bzw. nur einigen Agrarunternehmen und Bodenbesitzern zu Gute kommen. Die Dörfer und 
besonders auch die kleineren Städte sind hingegen aus dem Wirtschaftsraum herausgelöst und ringen ohne Funktion um ihre Zukunft, mit allen damit verbundenen Problemen wie Schrumpfungsprozessen und Sicherung der Daseinsvorsorge. Doch schaffen gerade diese Transformationsprozesse auch Räume für Veränderungen.

In eigenen Kurzbeiträgen oder in Form von Interviews stellen 24 Neulandgewinner auf etwa 200 Seiten ihre Projekte in Mecklenburg-Vorpommern (7), Sachsen-Anhalt (8), Brandenburg (5) und Sachsen (4) vor. Jeweils mit eindrucksvollen Fotos illustriert sowie durch eine Kurzcharakteristik eingeleitet und kartographisch eingeordnet, erhalten die Leser auf diese Weise sowohl gut strukturierte Übersichten als auch sehr anschauliche Schilderungen von den vielfältigen kreativen Projekten und Raumpionieren. Sichtbar werden persönliche Erfahrungen, Erfolge und auch Hürden. So finden sich auch zwei gescheiterte Vorhaben unter den Projekten. Je nach individueller Stärke, beruflichem und privatem Kontext umspannen die Projekte ein breites Themenspektrum gesellschaftlichen Zusammenlebens im ländlichen Raum, von der Erzeugung regionaler Produkte, Mobilität und Energieversorgung über Aufgaben des Denkmalschutzes bis zu Bildung, Kommunikation und Kultur sowie auch die Stärkung bürgerschaftlichen Engagements selbst. So entsteht beispielsweise in MecklenburgVorpommern eine „Werkstatt des guten Lebens“, in der sowohl alltägliche Probleme als auch Weltoffenheit und die Erzeugung regionaler Produkte eine Rolle spielen, in Brandenburg ein „Luftschloss 2.0“, das ein altes Bahngelände in ein soziales Zentrum für die Kreisstadt Neuruppin verwandeln soll, in Sachsen ein „Zukunfts-Projektor“, mit dem Bewusstseinsprozesse und kreative Lösungen für Leerstand im ländlichen Raum entwickelt werden sollen, und in Sachsen-Anhalt eine „Frische Küche“, welche die Essensversorgung für Kinder und Senioren wieder auf einen regionalen, nachhaltigen Weg bringen und gleichzeitig Bildung für gesündere Ernährung betreiben will - um nur einige Beispiele zu nennen. Zu den Neulandgewinnern gehören „Daheimgebliebene“, „Rückkehrer“ und auch „Städter“, die außerhalb der Metropolen in den ländlichen Regionen neue Freiräume finden; häufig spielt die (Um)Gestaltung von Gebäuden und Freiflächen eine zentrale Rolle.

Zur Gliederung der Neulandgewinner-Projekte in der $\mathrm{Pu}$ blikation legen die Herausgeber verschiedene Perspektiven an, wie die räumliche, Wissens-, zeitliche, Engagement-, Innen-, organisatorische, wirtschaftliche, Einkommens-, kommunalpolitische und gesellschaftspolitische Perspektive. Zwar erschließen sich diese Perspektiven teilweise erst bei etwas näherer Betrachtung, doch lenken sie den Blick auf wichtige Aspekte, die es zur Erforschung der gesellschaftlichen Transformationen sowie der Raumpioniere noch weiter auszuleuchten gilt. So wird beispielsweise die wirtschaftliche Perspektive zwar adressiert, doch bleibt diese letztlich offen und wird zugleich von verschiedenen Neulandgewinnern kritisch thematisiert. Dauerhaft können die zivilgesellschaftlichen Initiativen nicht allein ehrenamtlich ohne eine wirtschaftliche Perspektive getragen werden. Vielmehr sind hierzu neue Governance-Arrangements aus Zivilgesellschaft, Staat und privater Wirtschaft gefragt, für die es auch entsprechender Weichenstellungen durch die Politik bedarf.

Im Buch finden sich zudem erste fachliche Einschätzungen. In fünf eingearbeiteten Beiträgen betrachten Prozessbegleiter und -beobachter, zwei Soziologen (Cordula Kropp, Heinz Bude), ein Landschafts- und Raumplaner (Klaus Overmeyer), ein Finanzexperte (Lukas Beckmann) und eine Journalistin (Tina Veihelmann), die Neulandgewinner jeweils aus ihrem spezifischen Blickwinkel. Hiermit stellen sie wertvolle Kontexte zu aktuell bedeutsamen Forschungs- und gesellschaftspolitischen Themenfeldern her, wie die (inter-)nationale Forschungslandschaft zu sozialen Innovationen (Kropp; vgl. z. B. Howaldt/Butzin/ Domanski et al. 2014), Wandelprozesse und Raumpioniere bzw. „Raumunternehmen“ in urbanen Räumen (Overmeyer), wirtschaftsethische und stiftungsrelevante Aspekte (Beckmann), die (positive) Dialektik von Orten und Menschen sowie die potenziell zunehmende Bedeutung ländlicher Räume für Stadtmenschen (Veihelmann) und den Paradigmenwechsel der Transformationsforschung hin zur Beobachtung realer gesellschaftlicher Experimente (Bude).

Insgesamt ist diese Publikation damit ein sehr empfehlenswertes, anschauliches Buch zum Stöbern und Einlesen, das vielfältige Ideen und Impulse für die Entwicklung ländlicher Räume nicht nur für Ostdeutschland bietet (vgl. Willisch 2012). Zugleich ist es eine wahre Fundgrube bzw. ,Schatzkiste ' für weitere Forschungen zu gesellschaftlichen Transformationen. Denn die Neulandgewinner bieten mit ihren Projekten im ländlichen Raum ein noch weitgehend unbeleuchtetes und möglicherweise auch unterschätztes Pendant zur „kreativen Kraft der Städte“, die in der derzeitigen Transformationsdebatte im Vordergrund stehen. In diesem Sinne sei dem Buch gewünscht, dass es eine breite Leserschaft findet und sowohl Pioniere zum Nachahmen als auch Raum- und Landschaftsplaner ebenso wie Politiker und Wissenschaftler zu weiteren Entwicklungen und Forschungen anregt.

\section{Literatur}

Faber, K.; Oswalt, P. (Hrsg.) (2013): Raumpioniere in ländlichen Regionen. Neue Wege der Daseinsvorsorge. Leipzig. = Edition Bauhaus 35 .

Howaldt, J.; Butzin, A.; Domanski, D.; Kaletka, C. (Hrsg.) (2014): Theoretical Approaches to Social Innovation - A Critical Literature Review: A deliverable of the project: "Social Innovation: Driving Force of Social Change" (SI-Drive). Dortmund.

Links, C.; Volke, K. (Hrsg.) (2009): Zukunft erfinden. Kreative Projekte in Ostdeutschland. Berlin. 
WBGU - Wissenschaftlicher Beirat der Bundesregierung Globale Umweltveränderungen (2011): Hauptgutachten. Welt im Wandel. Gesellschaftsvertrag für eine Große Transformation. Zusammenfassung für Entscheidungsträger. Berlin.
Willisch, A. (Hrsg.) (2012): Wittenberge ist überall. Überleben in schrumpfenden Regionen. Berlin. 